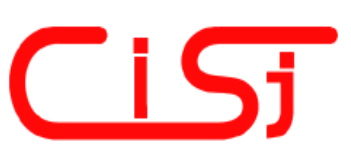

\title{
AN ITERATIVE METHOD FOR THE EVALUATION OF THE REGULARIZATION PARAMETER IN REGULARIZED IMAGE RESTORATION
}

\author{
Said E. El-Khamy ${ }^{1)}$, Mohiy M. Hadhoud ${ }^{3)}$, Moawad I. Dessouky ${ }^{2}$, \\ Bassiouny M. Salam ${ }^{2)}$, Fathi E. Abd El-Samie ${ }^{2)}$ \\ ${ }^{1)}$ Department of Electrical Engineering, Faculty of Engineering, \\ Alexandria University, Alexandria, 21544, Egypt \\ ${ }^{2)}$ Department of Electronics and Electrical Communications, Faculty of Electronic Engineering, \\ Menoufia University, 32952, Menouf, Egypt \\ ${ }^{3)}$ Department of Information Technology, Faculty of Computers and Information, \\ Menoufia University, 32511, Shebin Elkom, Egypt \\ E-mails: elkhamy@ieee.org, mmhadhoud@yahoo.com,dr_moawad@yahoo.com, \\ b_m_salam@yahoo.com and fathi_sayed@yahoo.com
}

\begin{abstract}
Regularized restoration is one of the powerful image restoration techniques because it preserves image details with a high degree of fidelity in the restored image. The main problem encountered in regularized image restoration is the evaluation of the regularization parameter. There are several methods for the evaluation of this parameter which require knowledge of the noise variance in the degraded image. After evaluating this parameter, regularized restoration is implemented by applying a regularization filter on the degraded image. In this paper, we propose a new iterative method for the evaluation of this parameter. This method depends on the maximization of the power in the restored image by the coincidence of the passband of the regularization filter with the frequency band in which, most of the image power exists. The suggested method doesn't require a priori knowledge of the noise variance. Results show that the estimated value of the regularization parameter leads to a minimum mean square restoration error.
\end{abstract}

Keywords: regularized restoration, regularization parameter, iterative regularization, inverse regularization.

\section{INTRODUCTION}

Digital image restoration is a problem which has been extensively treated in the literature [1-12]. The main objective of image restoration is to obtain a good estimate of the original image from a degraded image. Degradations in images have several origins such as out of focus blurring, linear motion blurring and Gaussian blurring. These types of blurring can be modeled as lowpass filters affecting the original image [13]. So, the image restoration problem is in fact a deconvolution problem. The existence of noise in the degraded image increases the difficulty of the image restoration process. The problem of deconvolution in the presence of noise is classified as an ill-posed inverse problem [6].

This problem has been solved in the literature using so many approaches [1-13]. In each approach, the mathematical basis on which the solution is based differs. In general, the purpose of image restoration is to obtain an estimate, which is as close as possible to the original image. One of the most popular approaches to the problem of image restoration is the linear minimum mean square error (LMMSE) restoration approach [13]. Rather than seeking a solution consistent with minimum contamination by noise, the LMMSE approach attacks the restoration problem directly and proposes a criterion that explicitly evaluates how close the restoration is to the original object intensity distribution. Despite being an easy solution, LMMSE restoration requires some assumptions related to the original unknown image and leads to some artifacts in flat areas of the restored images.

Regularization theory, which was basically introduced by Tikhonov and Miller has proved to be a good candidate for the solution of the image restoration problem [5]. There is no guarantee for the existence, uniqueness and stability of the solution of an inverse ill-posed problem like the image restoration problem based on direct inversion. So, there is a need for some constraints on the 
solution. The stabilizing functional approach is one of the basic methodologies for the development of regularized or constrained solutions. According to this approach, an ill-posed problem can be formulated as the constrained minimization of a certain functional, called the stabilizing functional [5].

For the implementation of regularized image restoration, there is a need to know both the regularization operator and the regularization parameter [1-12]. The rule of the regularization operator is to move the small Eigenvalues of the image degradation matrix away from zero while leaving the large Eigenvalues unchanged. It also incorporates prior knowledge about the required degree of smoothness of the restored image into the restoration process. The regularization parameter controls the trade-off between fidelity of the data and the smoothness of the solution [5, 6]. Hence, its determination is a very important issue. The evaluation of the regularization parameter can be performed using several techniques but most of these techniques are based on the amount of noise in the degraded image [6].

In this paper, we propose an iterative method for the evaluation of the regularization parameter based on the maximization of the total power content of the restored image. The rest of the paper is organized as follows. Section 2 gives the image degradation model. Section 3 discusses regularized image restoration. Section 4 surveys some existing methods for the evaluation of the regularization parameter. Section 5 presents the proposed method for the evaluation of the regularization parameter. Section 6 gives the experimental results. Finally, the concluding remarks are given in section 7 .

\section{IMAGE DEGRADATION MODEL}

Image restoration algorithms are, generally, designed to exploit characteristics of an image and its degradation. Accurate knowledge of the degradation is essential for any image restoration algorithm to be a successful algorithm. To obtain information about the degradation, we can gather information from the degraded image itself. As an example, if an image is blurred and we can identify a region in the degraded image where the original undegraded signal is known, we may be able to estimate the blurring function $h\left(n_{1}, n_{2}\right)$. The original image is denoted by $f\left(n_{1}, n_{2}\right)$, while the degraded image is denoted by $g\left(n_{1}, n_{2}\right)$. In the presence of noise $n\left(n_{1}, n_{2}\right)$, this can be represented as follows [1-13]:

$$
g\left(n_{1}, n_{2}\right)=h\left(n_{1}, n_{2}\right) * f\left(n_{1}, n_{2}\right)+n\left(n_{1}, n_{2}\right)
$$

If $f\left(n_{1}, n_{2}\right)$ is assumed to be known in some regions, then $g\left(n_{1}, n_{2}\right)$ and $f\left(n_{1}, n_{2}\right)$ can be used in these regions to estimate $h\left(n_{1}, n_{2}\right)$. This linear shift invariant image degradation model can be represented in another form as follows [1-13]:

$$
\mathbf{g}=\mathbf{H f}+\mathbf{n}
$$

where $\mathbf{f}, \mathbf{g}$ and $\mathbf{n}$ are lexicographic orders by either column or row of the $\mathrm{M} \times \mathrm{M}$ image, the degraded image and the noise, respectively. The matrix $\mathbf{H}$ is the discrete representation of the degradation of dimensions $\mathrm{M}^{2} \times \mathrm{M}^{2}$. For linear shift invariant systems, the matrix $\mathbf{H}$ is a block Toeplitz matrix. The objective of image restoration is to estimate $\mathbf{f}$ given the samples of the recorded image $\mathbf{g}$.

\section{REGULARIZED IMAGE RESTORATION}

According to the regularization theory, the solution of Eq. (2) is obtained by the minimization of the cost function [1-12]:

$$
\Psi(\mathbf{f})=\|\mathbf{g}-\mathbf{H} \mathbf{f}\|^{2}+\lambda\|\mathbf{C} \mathbf{f}\|^{2}
$$

where $\mathbf{C}$ is the regularization operator and $\lambda$ is the regularization parameter. This minimization is accomplished by taking the derivative of the cost function yielding [1-12]:

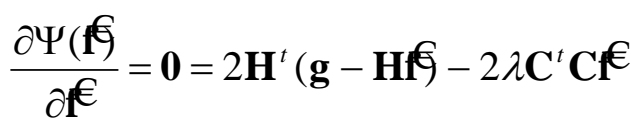

Solving for that $€$ that provides the minimum of the cost function gives [1-12]:

$$
€=\left(\mathbf{H}^{t} \mathbf{H}+\lambda \mathbf{C}^{t} \mathbf{C}\right)^{-1} \mathbf{H}^{t} \mathbf{g}=\mathbf{A}(\lambda) \mathbf{g}
$$

where

$$
\mathbf{A}(\lambda)=\left(\mathbf{H}^{t} \mathbf{H}+\lambda \mathbf{C}^{t} \mathbf{C}\right)^{-1} \mathbf{H}^{t}
$$

The regularization operator $\mathbf{C}$ incorporates prior knowledge about the required degree of smoothness of $\mathbf{f}$ into the restoration process. It can be a finite difference matrix chosen to minimize the second order or higher order difference energy of the estimated image [1]. The 2-D Laplacian is the best choice for this purpose. The rule of the regularization operator $\mathbf{C}$ is to move the small Eigenvalues of $\mathbf{H}$ away from zero while leaving the large Eigenvalues unchanged. The amount of change of these Eigenvalues is dependent on the choice of the regularization parameter $\lambda$.

Equation (5) can be written in an equivalent form using the Toeplitz to circulant approximation as follows [1]: 


$$
\oiint_{(u, v)}=\frac{H^{*}(u, v)}{|H(u, v)|^{2}+\lambda|C(u, v)|^{2}} G(u, v)=D(u, v, \lambda) G(u, v)
$$

where $F(u, v)$ is the Fourier transform of the original image, and $G(u, v), F(u, v), H(u, v), C(u, v)$ and $N(u, v)$ are the Fourier transforms of the degraded image, the estimate of the original image, the point spread function PSF of the blurring operator, the regularization operator and the noise, respectively. Thus, the transfer function of the regularization filter is given by [1]:

$$
D(u, v, \lambda)=\frac{H^{*}(u, v)}{|H(u, v)|^{2}+\lambda|C(u, v)|^{2}}
$$

If $\lambda=0$, this leads to the inverse filter solution defied as [1]:

$$
F(u, v)=\frac{G(u, v)}{H(u, v)}=F(u, v)+\frac{N(u, v)}{H(u, v)}
$$

In the presence of noise, the restoration problem has an ill-posed nature. Thus, severe deteriorations are observed in the restored images in the cases of low signal to noise ratios if the inverse filter solution is used. So, the second term in the denominator of Eq. (8) solves the ill-posed problem. This means that our problem is how to evaluate the regularization parameter $\lambda$.

\section{METHODS OF EVALUATING THE REGULARIZATION PARAMETER}

The problem of evaluating the regularization parameter has been addressed using a diversity of techniques $[6,7]$. Most of these techniques require knowledge of the noise variance of the degraded image $\sigma^{2}$. In many practical applications, the noise variance in the degraded image is not known and its estimation may be a tedious problem. We will survey some of these methods in the following subsections.

\subsection{CONSTRAINED LEAST SQUARES (CLS) METHOD}

In this method, the parameter $\lambda$ is selected such that the following equation is satisfied [6]:

$$
\|\mathbf{g}-\mathbf{H} \mathfrak{f}(\lambda)\|^{2}=\|(\mathbf{I}-\mathbf{H A}(\lambda)) \mathbf{g}\|^{2}=\|\mathbf{n}\|^{2}=\varepsilon^{2}=M^{2} \sigma^{2}
$$

Using the constrained least squares method is equivalent to assuming that the $\mathrm{i}^{\text {th }}$ component of the error $\mathbf{g}-\mathbf{H f}(\lambda)$ is Gaussian and $\|\mathbf{g}-\mathbf{H} \mathfrak{f}(\lambda)\|^{2}$ is Chi-square distributed with variance $\sigma^{2}$ and $M^{2}$ degrees of freedom.

\subsection{EQUIVALENT DEGREES OF FREEDOM (EDF) METHOD}

The notation of the equivalent degrees of freedom (EDF) can also be incorporated into the evaluation of the regularization parameter [6]. The EDF method takes into account the linear dependency between the degraded image $\mathbf{g}$ and the estimated image $\mathfrak{f}(\lambda)$. Therefore, $\|\mathbf{g}-\mathbf{H} \mathfrak{f}(\lambda)\|^{2}$ is Chi-square distributed with variance $\sigma^{2}$ and trace $(\mathbf{I}-\mathbf{H A}(\lambda))$ degrees of freedom. Thus, in this case, the constraint equation used for computing $\lambda$ will be given by [6]:

$$
\begin{aligned}
& \|\mathbf{g}-\mathbf{H} \hat{\mathbf{f}}(\lambda)\|^{2}=\|(\mathbf{I}-\mathbf{H A}(\lambda)) \mathbf{g}\|^{2}= \\
& \sigma^{2} \operatorname{trace}[\mathbf{I}-\mathbf{H A}(\lambda)]
\end{aligned}
$$

\subsection{MEAN SQUARE ERROR (MSE) METHOD}

Another method for evaluating $\lambda$ is obtained by directly minimizing the MSE function. The MSE function can be written as [6]:

$$
\begin{aligned}
& E\left[\|\mathbf{e}(\lambda)\|^{2}\right]=E\left[\|\mathbf{f}-\hat{\mathbf{f}}(\lambda)\|^{2}\right]= \\
& \|\mathbf{f}\|^{2}+E\left[\|\hat{\mathbf{f}}(\lambda)\|^{2}\right]-2 E\left[\mathbf{f}^{\mathbf{t}} \hat{\mathbf{f}}(\lambda)\right]
\end{aligned}
$$

Since the term $\|\mathbf{f}\|^{2}$ does not depend on $\lambda$, the minimization of $E\left[\|\mathbf{e}(\lambda)\|^{2}\right]$ is equivalent to the minimization of $E\left[\|\mathfrak{f}(\lambda)\|^{2}\right]-2 E\left[\mathbf{f}^{\mathbf{t}} \mathbf{f}(\lambda)\right]$. Using the Toeplitz to circulant approximation, we get [6]:

$$
E\left[\|\mathfrak{f}(\lambda)\|^{2}\right]=E\left[\sum_{i=1}^{M^{2}} \frac{\left|h_{i}\right|^{2}\left|G_{i}\right|^{2}}{\left(\left|h_{i}\right|^{2}+\lambda\left|c_{i}\right|^{2}\right)^{2}}\right]
$$

and

$$
\begin{aligned}
& E[\mathbf{f} \mathbf{t} \hat{\mathbf{f}}(\lambda)]=E\left[\sum_{i=1}^{M^{2}} \frac{\left|h_{i}\right|^{2}\left|F_{i}\right|^{2}}{\left(\left|h_{i}\right|^{2}+\lambda\left|c_{i}\right|^{2}\right)^{2}}\right]= \\
& E\left[\sum_{i=1}^{M^{2}} \frac{\left|G_{i}\right|^{2}-\sigma^{2}}{\left(\left|h_{i}\right|^{2}+\lambda\left|c_{i}\right|^{2}\right)^{2}}\right]
\end{aligned}
$$

where $h_{i}$ and $c_{i}$ are the Eigenvalues of $\mathbf{H}$ and $\mathbf{C}$, respectively, and $F_{i}$ and $G_{i}$ are the $\mathrm{i}^{\text {th }}$ components 
of the discrete Fourier transforms of $f$ and $g$, respectively.

Substituting Eqs. (13), (14) into Eq. (12) and letting the derivative of the MSE function equal to zero we get:

$$
\begin{aligned}
& \sum_{i=1}^{M^{2}} \frac{\lambda^{2}\left|c_{i}\right|^{4}\left|G_{i}\right|^{2}}{\left(\left|h_{i}\right|^{2}+\lambda\left|c_{i}\right|^{2}\right)^{3}}- \\
& \sigma^{2} \sum_{i=1}^{M^{2}} \frac{\lambda\left|c_{i}\right|^{2}}{\left(\left|h_{i}\right|^{2}+\lambda\left|c_{i}\right|^{2}\right)^{2}}=0
\end{aligned}
$$

In matrix form, this is equivalent to:

$$
\begin{gathered}
\left\|\mathbf{C}^{-1}[(\mathbf{I}-\mathbf{H A}(\lambda))]^{3 / 2} \mathbf{g}\right\|^{2}= \\
\sigma^{2} \text { trace }\left[\mathbf{C}^{-2}(\mathbf{I}-\mathbf{H A}(\lambda))^{2}\right]
\end{gathered}
$$

The solution of Eq. (16) leads to an estimate of the regularization parameter $\lambda$.

\section{4 PREDICTED MEAN SQUARE ERROR (PMSE) METHOD}

Another criterion for choosing the regularization parameter is based on the minimization of the weighted error norm [6]:

$$
E\left[\|\mathbf{H e}(\lambda)\|^{2}\right]=E\left[\|\mathbf{H f}-\mathbf{H f}(\lambda)\|^{2}\right]
$$

This method is based on the fact that the data points, which correspond to large Eigenvalues of $\mathrm{H}$ are more reliable, and thus they are weighted heavier. In matrix form, the regularization parameter $\lambda$ can be evaluated by solving the following equation [6]:

$$
\begin{aligned}
& \left.E\|\mathbf{H e}(\lambda)\|^{2}\right]=\|(\mathbf{I}-\mathbf{H A}(\lambda)) \mathbf{g}\|^{2}+ \\
& 2 \sigma^{2}\left[\operatorname{trace}[\mathbf{H A}(\lambda)]-M^{2}\right]
\end{aligned}
$$

\section{5 SET THEORETIC (ST) METHOD}

In this method, a priori knowledge about $\mathbf{f}$ is assumed which restricts the solution to lie in a set

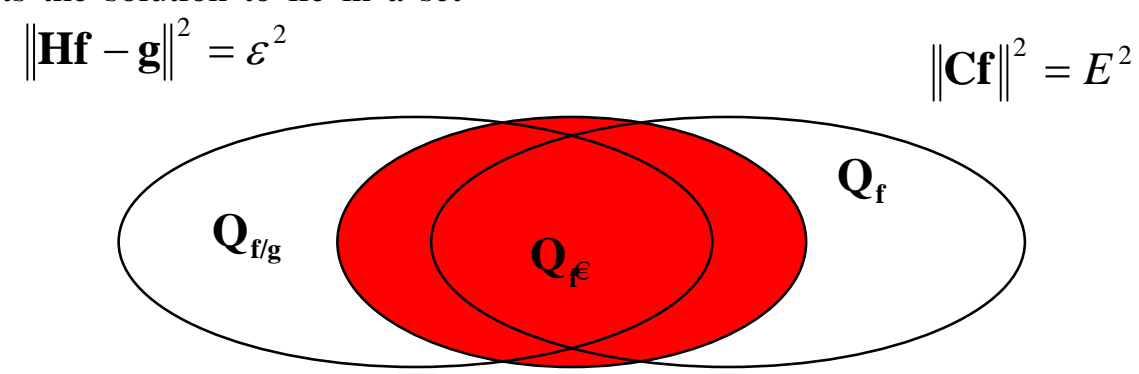

Fig. 1 - Geometric interpretation for the set theoretic approach using ellipsoids 


\section{THE SUGGESTED METHOD FOR EVALUATING THE REGULARIZATION PARAMETER}

The different methods mentioned above for evaluating the regularization parameter yield different estimates of $\lambda$. The regularization parameter $\lambda$ dictates, in general, the level of smoothness in the restored image. Thus, if two different regularization parameters $\lambda_{a}$ and $\lambda_{b}$ are used in restoring a degraded image with $\lambda_{a}>\lambda_{b}$, the resulting restored image $€\left(\lambda_{a}\right)$ will be smoother than $€\left(\lambda_{b}\right)$ [6]. The smoothness criterion doesn't reveal any thing about the MSE of the solution.

Based on the smoothness criterion, the smoothness of the solutions obtained using the different methods of evaluation differs according to the value of $\lambda$ obtained with each method. Some methods yield over smoothed estimates of the restored images while others yield under smoothed estimates [6]. Accordingly, a unified approach is needed to estimate the regularization parameter $\lambda$. We will now present the proposed method for evaluating the regularization parameter that can achieve minimum mean square restoration error.

The regularized image restoration filter transfer function is expressed in Eq. (8). It is clear from this equation that the parameter $\lambda$ controls the passband of this restoration filter. This is illustrated in Figs (2) to (6). Figure (2) illustrates the frequency response of a lowpass blurring operator $H(u, v)$. Figure (3) illustrates the frequency response of the inverse filter. It is clear that the inverse filter has singularities at certain frequencies. In Figs. (4) to (6), the effect of regularization is illustrated with a regularization parameter $\lambda=0.1,0.01$ and 0.001 , respectively. It is clear that the regularized filter has a bandpass nature and the parameter $\lambda$ controls the location of the passband in the frequency domain.

In general, most images to be restored using this regularized restoration filter have their spatial frequencies concentrated near the low frequency region of the spectrum. When the filter passband coincides with the spectral region at which the frequency contents of the image to be restored lie, good restoration results are obtained due to the preservation of all frequency contents of the restored image. The noise frequency components, which extend to infinite frequencies lie outside the filter passband and thus are reduced.

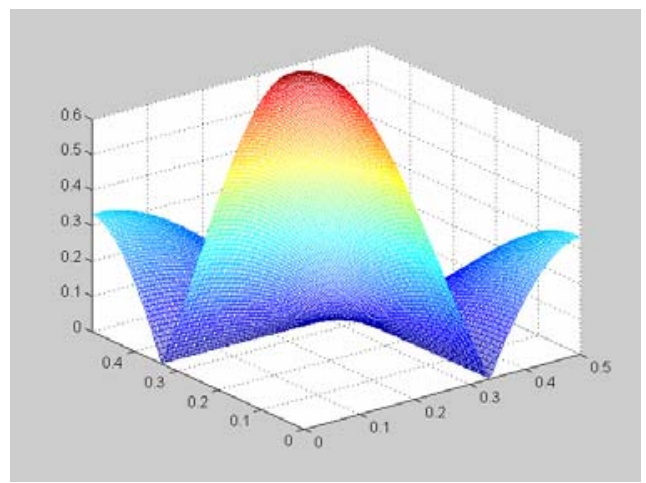

Fig. 2 - Low pass filter frequency response

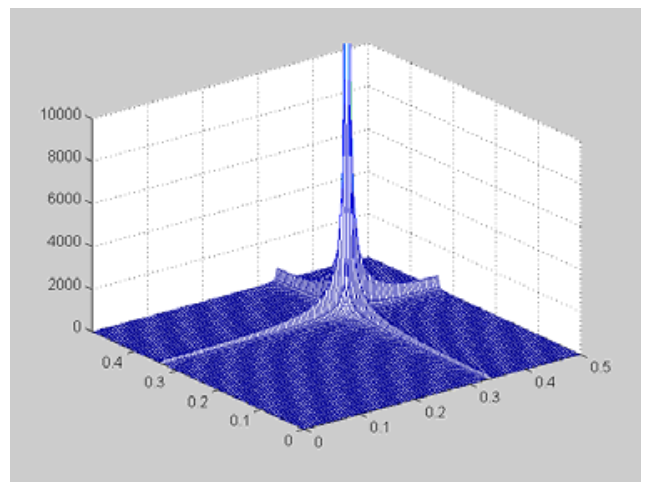

Fig. 3 - Inverse Filter frequency response

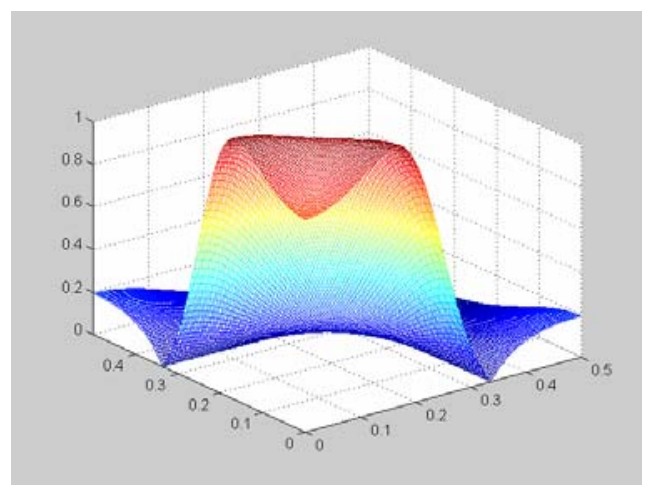

Fig. 4 - Regularized Filter frequency response $\lambda=0.1$

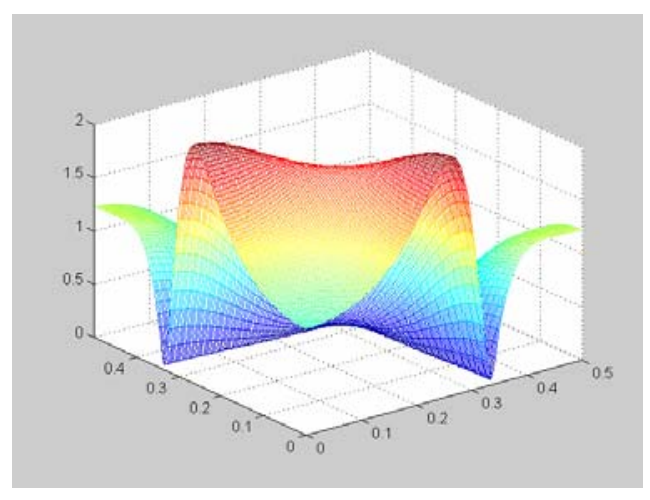

Fig. 5 - Regularized Filter frequency response $\lambda=0.01$ 


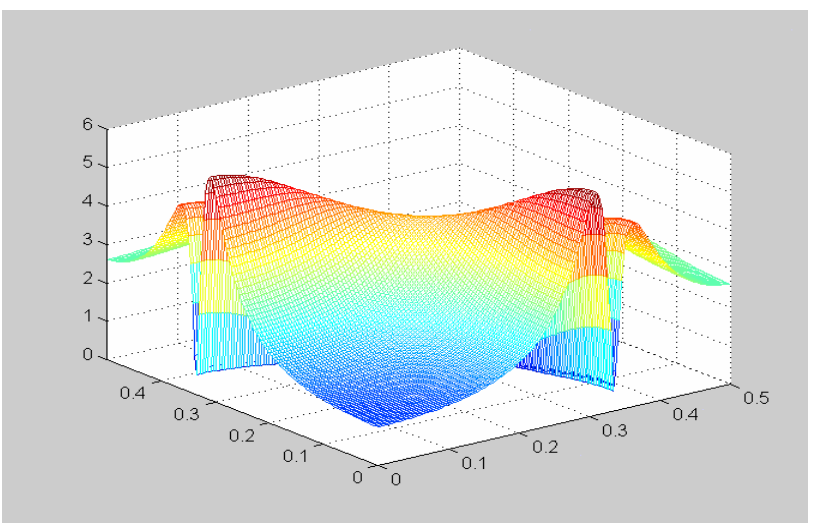

Fig. 6 - Regularized Filter frequency response $\lambda=0.001$

The suggested method for the evaluation of the regularization parameter is based on the abovementioned concept as illustrated in Fig. (7). In this method, an initial value for the regularization parameter is assumed and the restoration process is performed on the degraded image $g\left(n_{1}, n_{2}\right)$ using the regularized filter $D(u, v, \lambda)$. The power spectrum of the restored image is estimated and then the total power in the restored image is calculated. This process is repeated iteratively by updating the regularization parameter value until a maximum value of the total power is obtained in the restored image. The value of $\lambda$ which yields this maximum total power is the best estimate of $\lambda$ expected to yield minimum mean square error in the restored image.

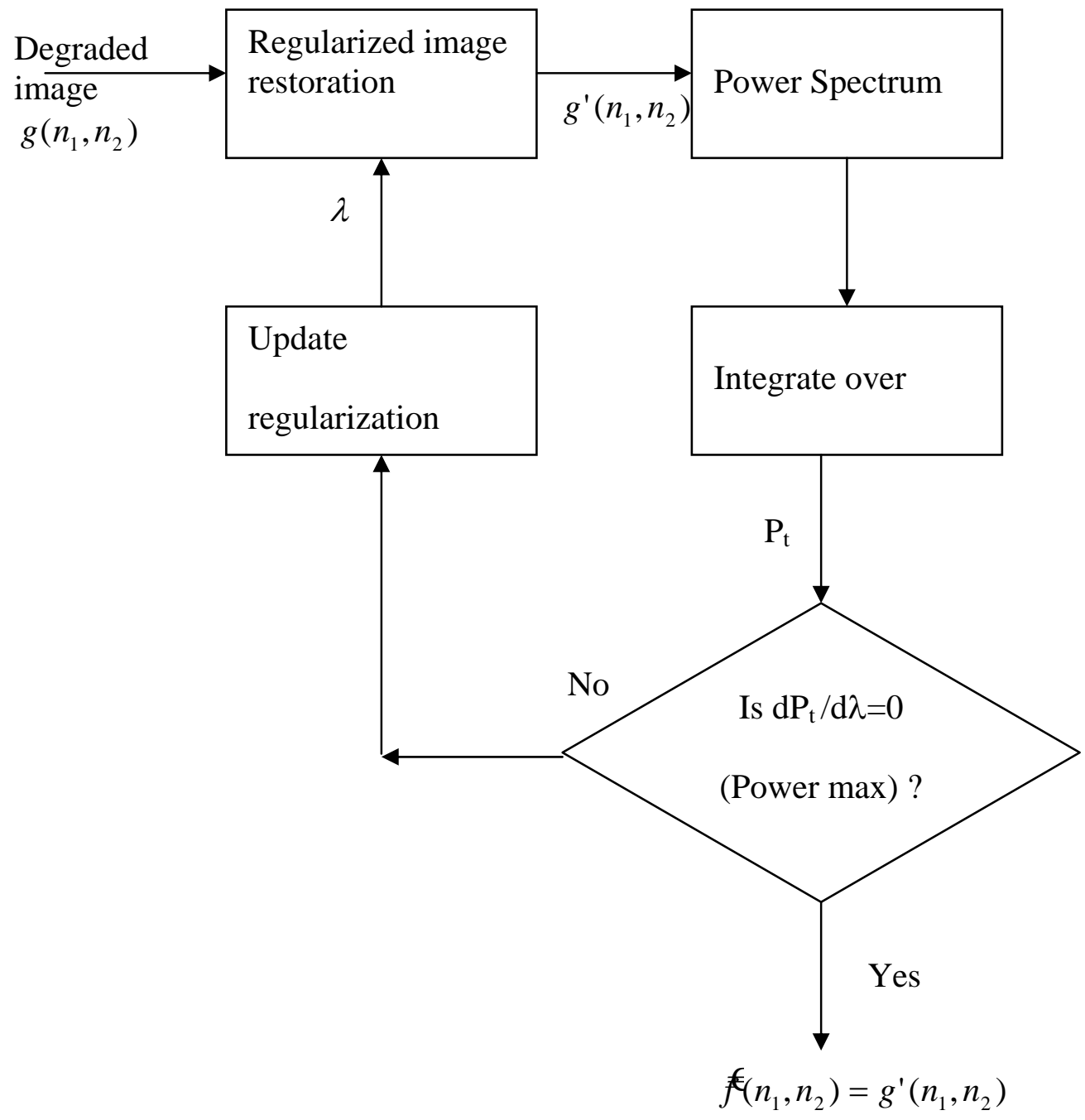

Fig. 7 - Block diagram of the suggested method for the evaluation of the regularization parameter

The auto-correlation function of the restored image $g^{\prime}\left(n_{1}, n_{2}\right)$ is evaluated using the following relation:

$$
R_{g^{\prime}}\left(n_{1}, n_{2}\right)=\frac{1}{w^{2}} \sum_{k=1}^{w} \sum_{l=1}^{w} g^{\prime}(k, l) g^{\prime}\left(n_{1}+k, n_{2}+l\right)
$$

where $w$ is an arbitrary window length.

The power spectrum of the restored image 
$g^{\prime}\left(n_{1}, n_{2}\right)$ is evaluated using the following relation:

$$
\left.P_{g^{\prime}}(u, v)=\sum_{n_{1}=0 n_{2}=0}^{N-1} \sum_{g^{\prime}}^{N-1} R_{1}, n_{2}\right) \exp \left\{-\frac{2 \pi \dot{d}}{N}\left(n_{1} u+n_{2} v\right)\right\}
$$

An integration process, which may be approximated using a summation process, is performed over the whole spectrum to evaluate the total power in the restored image $g^{\prime}\left(n_{1}, n_{2}\right)$. The approximation of this integration process is performed as follows:

$$
P_{t}=\frac{1}{N^{2}} \sum_{u}^{N-1} \sum_{v}^{N-1}\left|P_{g^{\prime}}(u, v)\right|
$$

When this total power is maximized, the restored image $g^{\prime}\left(n_{1}, n_{2}\right)$ can be considered the best estimate of the image $€\left(n_{1}, n_{2}\right)$. If the power is not maximum, the regularization parameter is updated and the process is repeated.

\section{SIMULATION RESULTS}

The suggested method for evaluating the regularization parameter is tested in this section for two different images with different spatial activities; the Cameraman and the Mandrill images illustrated in Figs. (8) and (9), respectively. The steps of the proposed method illustrated in Fig. (7) are applied on both the degraded Cameraman and Mandrill images.

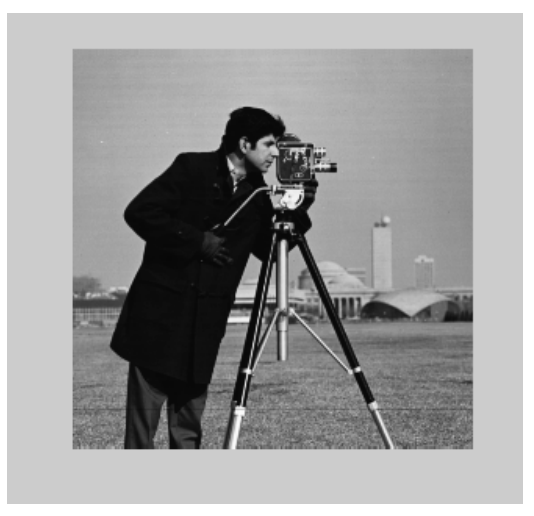

Fig. 8 - Original Cameraman image

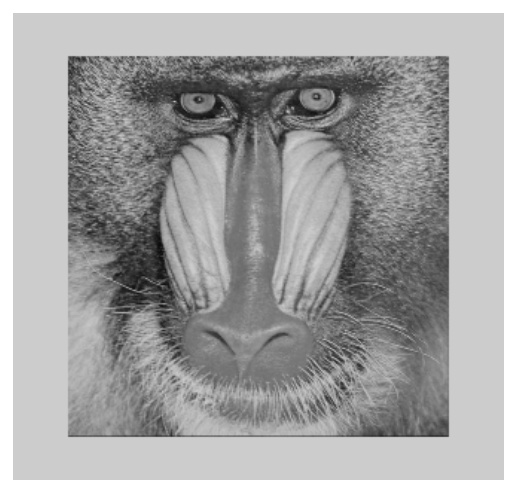

Fig. 9 - Original Mandrill image
The restoration process is performed in the presence of additive noise and a blurring operator of size $5 \times 5$ with an $\mathrm{SNR}=40 \mathrm{~dB}$. The variations of the total power and MSE in the restored images with the value of the regularization parameter are plotted in Figs. (10) and (12), respectively, for the Cameraman image and in Figs. (11) and (13), respectively, for the Mandrill image. It is clear that the minimum MSE coincides with the maximum power for the Cameraman image, which is mainly an image of low frequency nature. This means that the proposed algorithm have succeeded in evaluating the best value of the regularization parameter.

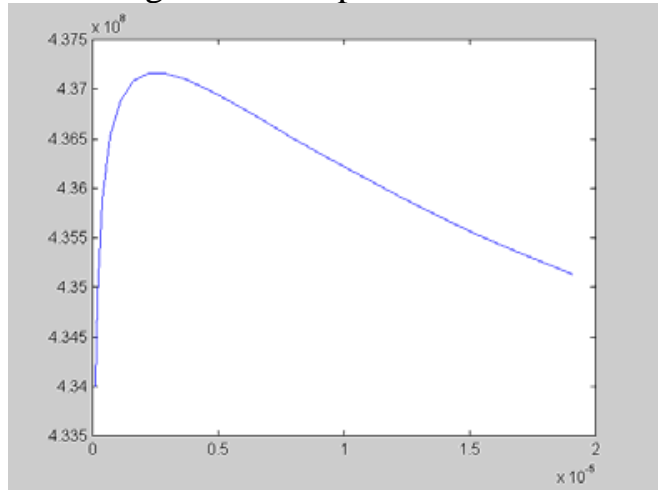

Fig. 10 - Pt versus $\lambda$ for restoring the Cameraman image degraded by $5 \mathrm{X} 5$ operator $\mathrm{SNR}=40 \mathrm{~dB}$

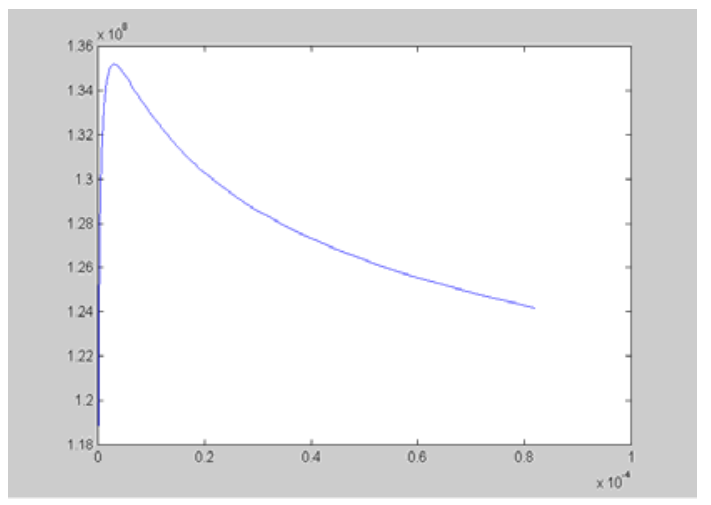

Fig. 11 - Pt versus $\lambda$ for restoring the Mandrill image degraded by $5 X 5$ operator $S N R=40 \mathrm{~dB}$

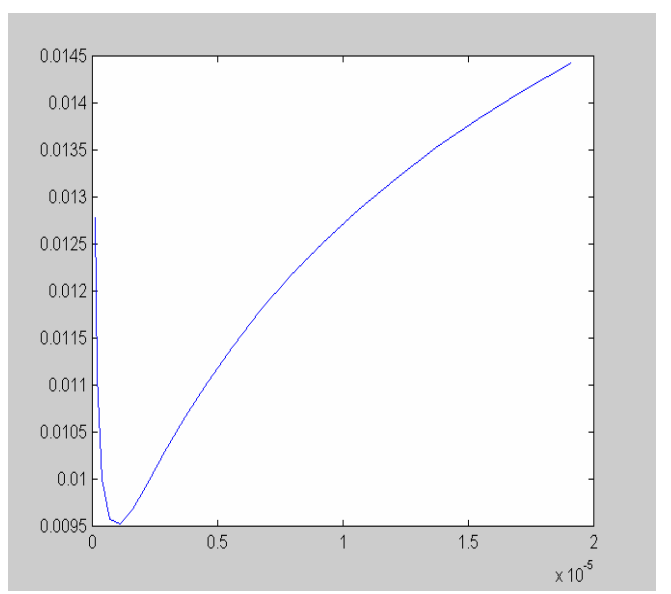

Fig. 12 - MSE versus $\lambda$ for restoring the Cameraman image degraded by $5 X 5$ operator $S N R=40 \mathrm{~dB}$ 


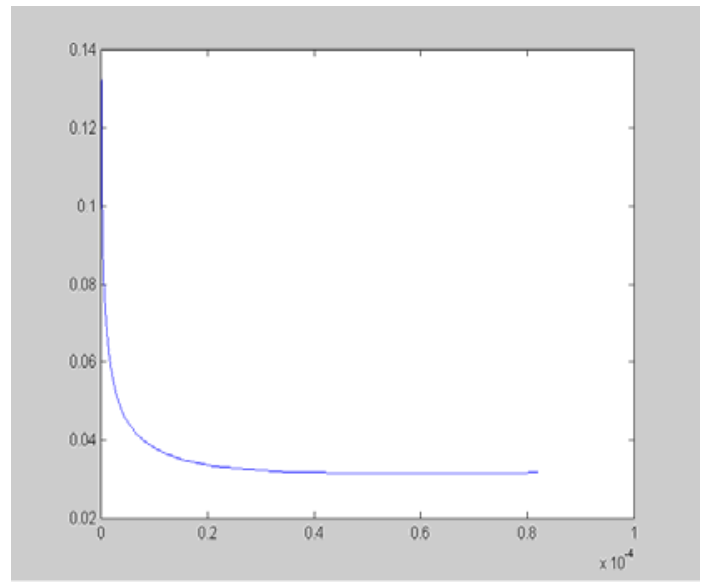

Fig. 13 - MSE versus $\lambda$ for restoring the Mandrill image degraded by $5 X 5$ operator $S N R=40 \mathrm{~dB}$

For the Mandrill image which is of a high frequency nature, the MSE reaches its minimum value with the maximum value of the total power in the restored image and keeps this minimum value for a long range of $\lambda$. This means that a wide range of $\lambda$ can be used for the restoration of images containing much high frequency details.

\section{CONCLUSIONS}

This paper has presented a new iterative method for the evaluation of the regularization parameter in regularized image restoration. This method has succeeded in evaluating the value of the regularization parameter which can maximize the power in the restored image without knowledge of the noise variance in the degraded image. The proposed method can be used in the restoration of images containing either low or high frequencies. For the restoration of images with low frequencies, a single value of the regularization parameter can be used, while for the restoration of images with high frequencies, a wide margin for the regularization parameter can be used.

\section{REFERENCES}

[1] H.C. Anderws and B.R. Hunt, Digital Image Restoration. Englewood Cliffs, NJ: PrenticeHall, 1977.

[2] W. K.Pratt, Digital Image Processing. John Wiley \& Sons, Inc., 1991.

[3] A. K.Jain, Fundamentals of Digital Image Processing. Prentice Hall, Inc, Englewood Cliffs, N.J., USA. 1989, reprinted in 1997.

[4] R. L. Lagendijk, J. Biemond and D. E. Boekee, "Regularized Iterative Image Restoration with Ringing Reduction”, IEEE Trans. Acoustics, Speech and Signal Processing, vol. 36, No.12, pp 1874-1888, Dec 1988.

[5] N. B. Karayiannis and A. N. Venetsanopoulos, "Regularization Theory in Image Restoration- the Stabilizing Functional Approach”, IEEE Trans. Acoustics, Speech and Signal Processing, Vol. 38, No.7, pp.1155-1179, July 1990.

[6] M.G. Kang and A. K. Katsagelos, "Simultaneous Iterative Image Restoration and Evaluation of the Regularization Parameter", IEEE Trans. Signal Processing, vol. 40, No.9,pp. 2329-2334 Sep. 1992.

[7] S. J. Receves and R. M. Mersereau, “Automatic Assessment of Constrained Sets in Image Restoration”, IEEE Trans. Image Processing, Vol. 1, No.1,pp.119-123, Jan. 1992.

[8] N. P. Galatsanos and A. K. Katasaggelos, “ Methods of Choosing the Regularization Parameter and Estimating the Noise Variance in Image Restoration and Their Relation”, IEEE Trans. Image Processing, Vol. 1, No.3, pp.322-336, July 1992.

[9] A. Seghouane, "A Note on Image Restoration Using Cp and MSE” IEEE Signal Processing Letters, Vol. 15, 2008.

[10] J. Xu and S. Osher, "Iterative Regularization and Nonlinear Inverse Scale Space Applied to Wavelet-Based Denoising” IEEE Transactions on Image Processing, Vol. 16, No. 2, 2007.

[11] J. Gutiérrez, F. J. Ferri, and J. Malo, "Regularization Operators for Natural Images Based on Nonlinear Perception Models” IEEE Transactions on Image Processing, Vol. 15, No. $1,2006$.

[12] M. R. Charest, Jr. and P. Milanfar, "On Iterative Regularization and its Application", IEEE Transactions on Circuits And Systems For Video Technology, Vol. 18, NO. 3, 2008.

[13] A. E. Savakis and H. J. Trussell, "Blur Identification by Residual Spectral Matching”, IEEE Trans. Image Processing, Vol. 2, No.2, pp.141-150, April 1993.

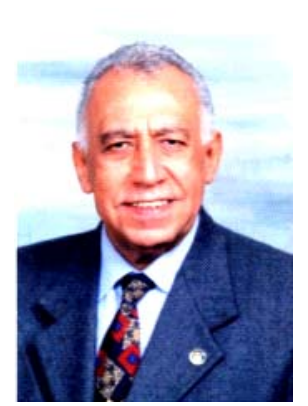

Said E. El-Khamy has received the B.Sc. (Hons) and M.Sc. degrees from Alexandria University, Alexandria, Egypt, in 1965 and 1967 respectively, and the Ph.D. degree from the University of Massachusetts, Amherst, USA. in 1971. He joined the teaching staff of the Department of Electrical Engineering, Faculty of Engineering, Alexandria University, Alexandria, Egypt, since 1972 and was appointed as a Full-time Professor in 1982 and as the Chairman of the Electrical Engineering Department since September 2000. His Current research areas of interest include Spread-Spectrum Techniques, Mobile and Personal Communications, 
Wave Propagation in different media, Smart Antenna Arrays, Space-Time Coding, Modern Signal Processing Techniques including Neural Networks, Wavelets, Genetic Algorithms, Fractals, HOS and Fuzzy Algorithms, and their applications in Image Processing, Communication Systems, Antenna design and Wave Propagation problems. He has published about two hundreds scientific papers in national and international conferences and journals. He took part in the organization of many local and international conferences including the yearly series of NRSC (URSI) series, ISCC'95, ISCC'97, ISSPIT'2000, MELECON'2002. He also chaired technical sessions in many local and international conferences including, ISSSTA'96, Mainz, Germany, Sept. 1966; IGARSS'98, Seattle, Washington, USA, July 1998 and AP-S'99, Orlando, Florida, USA, July 1999.

Prof. El-Khamy has earned many national and international research awards among which are the Alexandria University Research Award, 1979, the IEEE, R.W.P. King best paper award of the Antennas and Propagation Society of IEEE, in 1980, the Egypt's National Engineering Research award for two times in 1980 and 1989, respectively, the Egypt's State Science \& Art Decoration of the first class, 1981, the A. Schuman's-Jordan's award for Engineering Research in 1982, the Egypt's state Excellence Decoration of the first class in 1995, the IEEE Egypt' Section Award for Supervision of the Best Student Graduation Project in Communications and Networks in Egypt for the two successive years: 2000 and 2001. Recently, he received the State Scientific Excellence award in Engineering Sciences for 2002. He has also received the most cited paper award from Digital Signal Processing Journal in 2008. He is a member of the Electromagnetics Academy, a member and secretary of Egypt's national committee of URSI and is currently Egypt's National URSI Correspondent for Commission C. He is also a member of Tau Beta Pi, Eta Kappa Nu and Sigma Xi. He established the Alexandria/Egypt IEEE Subsection since 1999 and acts as its chairman since then.

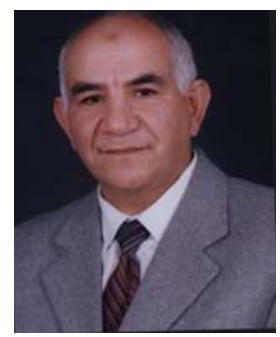

Mohiy M. Hadhoud received the $B S c$ and $M S c$ degrees in Electrical Engineering from Menoufia University in Egypt in 1976 and 1981 respectively. He received the $P h D$ degree from Southampoton University in 1987. He is currently a professor in the Dept. of Information Technology, Faculty of Computers and Information, Menoufia University. He has received the most cited paper award from Digital Signal Processing Journal in 2008. His areas of interests are signal processing, Image Processing and Digital Communications.

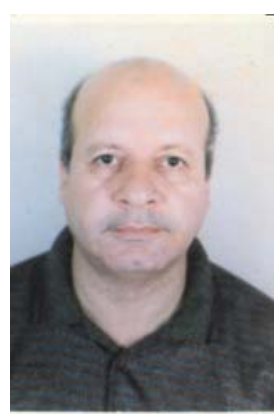

Moawad I. Dessouky received the BSc and MSc degrees in Electrical Engineering from Menoufia University in Egypt in 1976 and 1981 respectively. He received the $P h D$ degree from McMaster University in 1986. He is currently a professor in the Dept. Electronics and Electrical Communications, Faculty of Electronic Engineering, Menoufia University. He has received the most cited paper award from Digital Signal Processing Journal in 2008. His areas of interests are signal processing, Image Processing and Digital Communications.

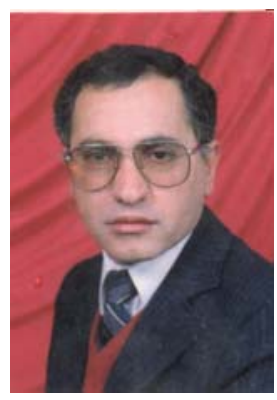

Bassiouny M. Sallam received the BSc degree in Electrical Engineering from Menoufia University and MSc degree from Cairo University. He received the $\mathrm{PhD}$ degree from Drexel University in USA. He is currently working with the Dept. Electronics and Electrical Communications, Faculty of Electronic Engineering, Menoufia University. He has received the most cited paper award from Digital Signal Processing Journal in 2008. His areas of interests are signal processing, Image Processing and Digital Communications.

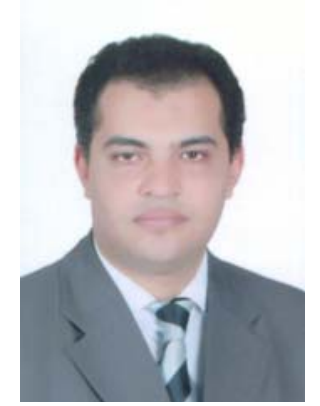

Fathi E. Abd El-Samie received the $B S c$ and $M S c$ and $P h D$ degrees in Electrical Engineering from Menoufia University in Egypt in 1998, 2001 and 2005, respectively. He is currently a lecturer in the Dept. of Electronics and Electrical Communications, Faculty of Electronic Engineering, Menoufia University. He has received the most cited paper award from Digital Signal Processing Journal in 2008. His areas of interests are signal processing, Image enhancement, restoration, super resolution and interpolation. 